\title{
Atividade inseticida in vitro do óleo de sementes de nim sobre Lutzomyia longipalpis (Diptera: Psychodidae)
}

\author{
In vitro insecticidal activity of seed neem oil on Lutzomyia longipalpis (Diptera: Psychodidae) \\ Michelline V. Maciel ${ }^{1}$; Selene M. Morais ${ }^{2}$; Claudia M.L. Bevilaqua ${ }^{1 *}$; Rafaella A. Silva ${ }^{1}$; Renata S. Barros ${ }^{1}$; \\ Raimundo N. Sousa ${ }^{3}$; Lindemberg C. Sousa ${ }^{3}$;yeghyna K.A. Machado ${ }^{2}$ Edy S. Brito ${ }^{4}$; Manoel A. Souza-Neto ${ }^{4}$ \\ ${ }^{1}$ Programa de Pós-Graduação em Ciências Veterinárias, Laboratório de Doenças Parasitárias, Universidade Estadual do Ceará - UECE \\ ${ }^{2}$ Laboratório de Química em Produtos Naturais, Universidade Estadual do Ceará - UECE \\ ${ }^{3}$ Laboratório de Entomologia, Núcleo de Controle de Vetores, Secretaria de Saúde do Estado do Ceará - SES/CE \\ ${ }^{4}$ Embrapa Agroindústria Tropical
}

Recebido 22 de Abril de 2009

Aceito em 13 de Julho de 2009

\section{Resumo}

Lutzomyia longipalpis é o principal vetor da Leishmaniose visceral no Brasil. O objetivo deste trabalho foi avaliar o efeito do óleo de sementes de nim (Azadirachta indica) sobre ovos, larvas e adultos do vetor. Os insetos foram capturados no campo e mantidos no laboratório a $\pm 27^{\circ} \mathrm{C} \mathrm{e} 80 \%$ de umidade relativa. Cinco tratamentos com diferentes concentraçôes foram realizados, usando-se dois controles negativos, um com água destilada e outro com Tween 80 (3\%) e um controle positivo com cipermetrina. Os ovos foram borrifados com o óleo em diferentes concentraçóes e avaliou-se o número de larvas eclodidas por 10 dias consecutivos. A mortalidade das larvas foi observada até a pupação, e a mortalidade dos adultos foi observada após 24, 48, e 72 horas. A análise estatística foi feita pelo teste de Tukey a $5 \%$ de probabilidade. No teste com ovos, a maior concentraçáo obteve $65,16 \pm 3,24 \%$ de eficácia. O teste com larvas apresentou $67,75 \pm 2,21 \%$ de eficácia na concentração de $100 \mathrm{mg} \cdot \mathrm{mL}^{-1}$. Com adultos, a eficácia na concentração de $100 \mathrm{mg} \cdot \mathrm{mL}^{-1}$ foi de $96,64 \pm 4,11 \%$, após 24 horas. A análise fitoquímica revelou a presença de triterpenos. Esses resultados demonstram o potencial uso desse óleo no controle deste vetor.

Palavras-chave: nim, flebotomíneos, óleo, leishmaniose, inseticidas botânicos.

\begin{abstract}
Lutzomyia longipalpis is the main vector of visceral leishmaniasis in Brazil. The objective was to evaluate the effect of oil from (Azadirachta indica) neem seeds on eggs, larvae and adults of the vector. The insects were captured in the field and kept in the laboratory at $\pm 27^{\circ} \mathrm{C}$ and $80 \%$ relative humidity. Five treatments with different concentrations were performed using two negative controls (distilled water and Tween 80 ) and a positive control. The eggs were sprayed with the oil at different concentrations and the number of hatched larvae evaluated for 10 days. Mortality of larvae was observed to pupation and adult mortality was observed after 24,48 , and 72 hours. Statistical analysis was performed by Tukey test at $5 \%$ probability. The highest oil concentration of eggs obtained $65.16 \pm 3.24 \%$ efficacy for reducing egg hatching. The test with larvae showed $67.75 \pm 2.21 \%$ efficacy at a concentration of $100 \mathrm{mg} \cdot \mathrm{mL}^{-1}$. In adults, the efficacy of the $100 \mathrm{mg} \cdot \mathrm{mL}^{-1}$ concentration was $96.64 \pm 4.11 \%$ after 24 hours. The phytochemical analysis revealed the presence of triterpenes. These results demonstrate the potential use of this oil in the control of this vector.
\end{abstract}

Keywords: neem, sandflies, oil, leishmaniasis, botanical insecticides.

\section{Introduçáo}

As leishmanioses estão entre as principais doenças causadas por agentes patogênicos transmitidos por vetores, sendo a Leishmaniose Visceral (LV) considerada a forma mais severa (SINGH, 2006).

\footnotetext{
*Autor para correspondência: Claudia Maria Leal Bevilaqua Programa de Pós-Graduação em Ciências Veterinárias,

Laboratório de Doenças Parasitárias, Universidade Estadual do Ceará - UECE

Av. Paranjana, 1.700, CEP 60.740-000, Fortaleza, Ceará, Brasil;

e-mail: claudiamlb@yahoo.com.br
}

Com a expansão da área de abrangência da doença e o aumento significativo do número de casos, a LV passou a ser considerada pela Organizaçáo Mundial de Saúde uma das prioridades dentre as doenças tropicais (GONTIJO; MELO, 2004). A transmissão é feita por vetores pertencentes à família Psychodidae, gênero Lutzomyia, sendo o principal vetor, no Brasil, Lutzomyia longipalpis (DESJEUX, 2004). O padrão de transmissão da doença pode-se modificar em virtude das alteraçóes ambientais e, consequentemente, 
à adaptação do vetor, associado às migraçôes da população (BEJARANO et al., 2002).

As estratégias de controle das doenças transmissíveis por vetores biológicos são complexas, principalmente quando associadas à existência de reservatórios domésticos e silvestres e aos aspectos ambientais. $\mathrm{O}$ uso de inseticidas tem sido direcionado, principalmente, contra as formas adultas do inseto vetor (GONTIJO; MELO, 2004). A resistência adquirida e a poluição ambiental, causadas pela aplicação repetida de inseticidas sintéticos persistentes, têm levado a um aumento no interesse por novos produtos químicos (VIEGAS-JÚNIOR, 2003). Além disso, o custo para o desenvolvimento de inseticidas químicos é alto e tem aumentado ao longo dos anos, por causa da necessidade de novas moléculas e formulaçóes mais adequadas, o que tem feito crescer o interesse pela pesquisa de inseticidas alternativos (ALMEIDA, 2001).

A utilização de plantas ou seus extratos com atividade inseticida tem aplicação importante na saúde pública e na agropecuária (MATIAS et al., 2002). O Brasil é o país com maior riqueza botânica, com 56 mil espécies de plantas das 256 mil existentes no mundo. Possui, ainda, um grande potencial como fonte de compostos biologicamente ativos provenientes de plantas medicinais. Plantas da família Meliaceae são conhecidas por conter uma variedade de compostos descritos como inseticidas, antialimentares e reguladores do crescimento (NAKATANI et al., 2004). Dentro dessa família, destaca-se a Azadirachta indica, conhecida por nim, que é considerada uma das mais importantes, graças à sua atividade sistêmica, eficiência em baixas concentraçôes e baixa toxicidade aos mamíferos (GALLO et al., 2002). O principal composto extraído dos frutos dessa planta é a azadiractina, um limonoide que atua interferindo no funcionamento de glândulas endócrinas que controlam a metamorfose em insetos e também apresenta propriedade fagoinibidora (VIEGAS-JÚNIOR, 2003). Além disso, na agricultura, muitas pesquisas têm sido realizadas, especialmente nas regióes tropicais, utilizando metabólitos secundários de plantas com a finalidade de proteger a colheita e armazenamento de grãos dos insetos. E uma das plantas mais estudadas é a $A$. indica (BOEKE et al., 2004)

Nesse contexto, o objetivo deste trabalho foi avaliar a atividade inseticida do óleo das sementes de $A$. indica sobre as três fases de desenvolvimento de L. longipalpis em laboratório.

\section{Material e Métodos}

\section{Estabelecimento da colônia de L. longipalpis}

O estabelecimento e a manutenção da colônia de flebotomíneos foram baseados em Smith (1925) apud Sherlock, I. A. e Sherlock, V. A. (1972) com algumas modificaçôes. Os insetos adultos foram capturados por meio de armadilhas do tipo CDC e encaminhados para o Laboratório de Entomologia do Núcleo de Endemias do Estado do Ceará (NUVET). Foram mantidos em gaiolas teladas, em BOD, à temperatura de $27^{\circ} \mathrm{Ce} 80 \%$ de umidade relativa. Os adultos foram alimentados, utilizando-se hamsters anestesiados (10 mg. $\mathrm{kg}^{-1}$ de ketamina e $2 \mathrm{mg} \cdot \mathrm{kg}^{-1}$ de xilazina por via intramuscular). Após 4 dias, as fêmeas foram inseridas com o auxílio de um capturador de Castro em potes plásticos $(4 \times 4,5 \mathrm{~cm})$ revestidos com gesso, previamente umedecidos com água destilada para postura. Uma vez realizada a postura, os ovos foram transferidos para novos potes plásticos revestidos com gesso, os quais foram acondicionandos em uma cuba forrada com areia umedecida, por 4 dias. Após o surgimento das larvas, adicionou-se o substrato para alimentação, composto por fezes de coelho e folhas trituradas de mandioca, até o período de pupação. Os adultos foram liberados nas gaiolas, dando continuidade ao ciclo. Os hamsters utilizados nesse trabalho foram provenientes do biotério do Laboratório de Entomologia da Secretaria de Saúde do Estado do Ceará, sendo fornecida água e ração à vontade, com acompanhamento Médico Veterinário. Este trabalho foi aprovado pelo Comitê de Ética para Uso de Animais (CEUA) da Universidade Estadual do Ceará, sob protocolo $\mathrm{n}^{\circ}$ 07.227.503-0.

\section{Obtenção do óleo da planta}

As sementes da planta foram coletadas no sítio Neem, localizado no município de Eusébio, Ceará. Para a obtenção do óleo, as sementes foram trituradas em uma prensa e o óleo coletado in natura.

\section{Análise fitoquímica do óleo}

Os testes fitoquímicos do óleo para fenóis, taninos, esteroides, triterpenoides e alcaloides foram realizados seguindo a metodologia descrita por Matos (1997), que investigaram os tipos de metabólitos secundários presentes nos extratos de plantas. Estes se baseiam na adição de determinados reagentes à solução hidroalcoólica do óleo da planta e a observaçáo das mudanças de cor e formaçáo de precipitados. No teste para esteroides e triterpenóides (Lieberman-Burchard), foi adicionado $1 \mathrm{~mL}$ de anidrido acético e, em seguida, com agitação, três gotas de ácido sulfúrico $\left(\mathrm{H}_{2} \mathrm{SO}_{4}\right)$ concentrado à solução de diclorometano do óleo. Coloração azul evanescente seguida de verde permanente é indicativa da presença de esteróides. Coloração parda até vermelha indica a presença de triterpenoides.

\section{Bioensaio sobre ovos}

Os ensaios realizados com ovos, larvas e adultos foram constituídos por cinco tratamentos com diferentes concentraçóes do óleo de $A$. indica, realizados à temperatura de $27^{\circ} \mathrm{C}$ e $80 \%$ de umidade relativa e acompanhados por controles negativos com água destilada e com o diluente Tween 80 a 3\%, e controle positivo com $196 \mu \mathrm{g} . \mathrm{mL}^{-1}$ de cipermetrina. Para cada concentração testada foram utilizadas três repetiçôes e três réplicas. Em cada repetição foram utilizados 30 ovos borrifados com $1 \mathrm{~mL}$ do óleo. Os potes contendo ovos foram cobertos com tecido scarlini até o aparecimento das larvas de L. longipalpis. As concentraçôes utilizadas foram: 6,12; 12,$5 ; 25 ; 50 \mathrm{mg}$ e $100 \mathrm{mg} \cdot \mathrm{mL}^{-1}$. Três dias após o tratamento com os óleos, iniciou-se a avaliação do número de larvas presentes em cada pote/concentração, por 10 dias consecutivos.

\section{Bioensaio com larvas}

O teste com larvas foi realizado nas mesmas condiçóes descritas para os ovos. Potes contendo 40 ovos de L. longipalpis foram acondicionados adequadamente até o surgimento das larvas 
contadas 6 dias pós-eclosão dos ovos. Em seguida, foi feita a borrifação dos potes com as soluçóes dos óleos. Cada repetição constituiu-se de 30 larvas. As concentraçôes utilizadas neste teste foram as mesmas descritas no bioensaio anterior. As larvas foram observadas até a pupação.

\section{Ensaio com adultos}

O ensaio foi realizado com 30 insetos adultos por pote, sendo 15 machos e 15 fêmeas; quatro dias após o repasto; os potes foram previamente borrifados com a solução do óleo de $A$. indica nas concentraçóes utilizadas anteriormente. Neste experimento, os parâmetros observados foram: mortalidade dos insetos às 24, 48 e 72 horas de exposição ao óleo, o número de ovos obtidos e o número de larvas eclodidas.

\section{Análise dos dados}

Os dados foram transformados pela fórmula: $\log (\mathrm{x}+1)$, submetidos à análise de variância one-way e comparados pelo teste de Tukey, com 5\% de probabilidade, usando-se o programa Prism 3.0. A concentração efetiva para eliminar 50\% dos insetos $\left(\mathrm{CE}_{50}\right)$ foi calculada pelo método de Probito usando o programa SPSS 8.0 para Windows.

\section{Resultados}

Os testes fitoquímicos revelaram no óleo de sementes a presença de triterpenos. Os resultados do teste com ovos e larvas do inseto estão dispostos na Tabela 1. No teste com ovos, o óleo apresentou eficácia de 65,16 $\pm 3,24 \%$ na maior concentração. Na concentração de $12,5 \mathrm{mg} \cdot \mathrm{mL}^{-1}$ ou inferior não se observou efeito ovicida. $\mathrm{A} \mathrm{CE}_{50}$ desse óleo foi de 35,44 (26,29 - 50,33) mg.mL $\mathrm{mL}^{-1}$. No teste com larvas o óleo apresentou eficácia de $67,75 \pm 2,21 \%$ na maior concentração. A CE ${ }_{50}$ desse óleo foi 60,98 $\left(45,93\right.$ - 91,62) mg. $\mathrm{mL}^{-1}$.

Os resultados do teste com insetos adultos estáo descritos na Tabela 2. O referido óleo apresentou eficácia de 96,64 \pm 4,11\% na concentração mais elevada após 24 horas de exposição. Não foram observadas variaçóes na eficácia adulticida em relação ao tempo. A $\mathrm{CE}_{50}$ do óleo foi de 33,73 $\left(18,87\right.$ - 73,18) mg.mL $\mathrm{mL}^{-1}$. Não houve diferença estatística em relação à mortalidade de machos e fêmeas do inseto $(\mathrm{p}>0,05)$. Com relação ao número e taxa de eclosão de ovos obtidos a partir de fêmeas expostas à ação do óleo de $A$. indica, entre o grupo tratado e controle, a diferença náo foi significativa $(\mathrm{p}>0,05)$. Em todos os ensaios, os resultados apresentaram efeito dose-dependente.

\section{Discussáo}

A leishmaniose visceral (LV) é uma doença reemergente, que apresenta expansão geográfica e processo de urbanização em várias regióes do Brasil (DANTAS-TORRES et al., 2006). Medidas de controle da LV, em vários países, têm sido associadas ao controle do vetor. $\mathrm{O}$ uso de dicloro-difenil-tricloroetano (DDT) residual na Índia para controlar malária resultou em uma
Tabela 1. Percentual de eficácia do óleo da semente de Azadirachta indica sobre ovos de Lutzomyia longipalpis, após 10 dias de observaçáo, e sobre larvas observadas até a pupação

\begin{tabular}{lcc}
\hline \multirow{2}{*}{ Concentraçáo } & \multicolumn{2}{c}{ \% Eficácia } \\
\cline { 2 - 3 } & \multicolumn{1}{c}{ Ovos } & Larvas \\
\hline 100 & $65,16 \pm 3,24^{\mathrm{a}}$ & $67,75 \pm 2,21^{\mathrm{a}}$ \\
50 & $30,36 \pm 3,29^{\mathrm{c}}$ & $36,38 \pm 2,71^{\mathrm{c}}$ \\
25 & $2,38 \pm 2,31^{\mathrm{d}}$ & $24,73 \pm 1,91^{\mathrm{d}}$ \\
12,5 & $0,0 \pm 0,0^{\mathrm{d}}$ & $9,74 \pm 3,07^{\mathrm{e}}$ \\
6,12 & $0,0 \pm 0,0^{\mathrm{d}}$ & $0,0 \pm 0,0^{\mathrm{f}}$ \\
Água & $0,14 \pm 0,44^{\mathrm{d}}$ & $0,0 \pm 0,0^{\mathrm{f}}$ \\
Tween $80(3 \%)$ & $0,0 \pm 0,0^{\mathrm{d}}$ & $0,0 \pm 0,0^{\mathrm{f}}$ \\
Cipermetrina $\left(196 \mu \mathrm{g} \cdot \mathrm{mL}^{-1}\right)$ & $100,0 \pm 0,0^{\mathrm{b}}$ & $100,0 \pm 0,0^{\mathrm{b}}$ \\
\hline
\end{tabular}

Letras minúsculas como expoente, quando diferentes na mesma coluna, indicam diferença significativa.

Tabela 2. Percentual de eficácia do óleo da semente de Azadirachta indica sobre adultos de Lutzomyia longipalpis após 24, 48 e 72 horas de observação.

\begin{tabular}{lccc}
\hline \multirow{2}{*}{ Concentraçáo } & \multicolumn{3}{c}{ \% Eficácia } \\
\cline { 2 - 4 } & 24 horas & 48 horas & 72 horas \\
\hline 100 & $96,64 \pm 4,11^{\mathrm{aA}}$ & $96,64 \pm 3,51^{\mathrm{aA}}$ & $96,47 \pm 3,70^{\mathrm{aA}}$ \\
50 & $51,33 \pm 6,94^{\mathrm{cA}}$ & $52,53 \pm 4,79^{\mathrm{cA}}$ & $47,64 \pm 4,68^{\mathrm{cA}}$ \\
25 & $33,70 \pm 2,21^{\mathrm{dA}}$ & $34,01 \pm 4,07^{\mathrm{dA}}$ & $25,80 \pm 5,45^{\mathrm{dA}}$ \\
12,5 & $19,48 \pm 2,14^{\mathrm{eA}}$ & $15,51 \pm 4,00^{\mathrm{eA}}$ & $9,44 \pm 4,18^{\mathrm{eA}}$ \\
6,12 & $4,10 \pm 2,95^{\mathrm{fA}}$ & $2,22 \pm 2,05^{\mathrm{fA}}$ & $2,96 \pm 3,21^{\mathrm{fA}}$ \\
Água & $0,49 \pm 0,80^{\mathrm{fA}}$ & $2,22 \pm 0,30^{\mathrm{fA}}$ & $1,36 \pm 1,70^{\mathrm{fA}}$ \\
Tween $80(3 \%)$ & $0,37 \pm 0,56^{\mathrm{fA}}$ & $2,22 \pm 0,86^{\mathrm{fA}}$ & $0,95 \pm 2,20^{\mathrm{fA}}$ \\
Cipermetrina & $100,0 \pm 0,0^{\mathrm{bA}}$ & $100,0 \pm 0,0^{\mathrm{bA}}$ & $100,0 \pm 0,0^{\mathrm{bA}}$ \\
$\left(196 \mu \mathrm{g} \cdot \mathrm{mL}^{-1}\right)$ & & &
\end{tabular}

Letras minúsculas como expoente, quando diferentes na mesma coluna, indicam diferença significativa.

Letras maiúsculas como expoente, quando diferentes em diferentes colunas, indicam diferença significativa

diminuição significante no número de casos da LV indicando que um controle efetivo pode ser alcançado. No entanto, faz-se necessário o desenvolvimento de novos inseticidas para o controle do vetor como também de um monitoramento das áreas borrifadas para avaliação de eficácia desses produtos, (COSTA, 2008). A necessidade de métodos mais eficazes e cada vez mais seguros no controle de insetos tem estimulado a busca de novos inseticidas em plantas (VIEGAS-JÚNIOR, 2003). Estudos recentes têm estimulado a investigação sobre propriedades de compostos químicos derivados de plantas e concluíram que eles são seguros, degradáveis e alvo-específicos (TARE et al., 2004).

Nicotina (Nicotiana tabacum), piretrinas (Tanacetum cinerariifolium) e rotenona (Derris spp e Lonchocarpus spp) são bons exemplos de substâncias usadas para controlar pragas agrícolas. Nesse contexto, as azadiractinas pertencentes à planta $A$. indica, uma árvore conhecida popularmente como nim, tem merecido destaque (PASCUAL-VILLALOBOS; ROBLEDO, 1998).

A eficácia do óleo de sementes de $A$. indica sobre três fases de desenvolvimento de $L$. longipalpis foi avaliada, demonstrando atividade inseticida sobre todas as fases testadas. No que se refere à atividade ovicida, resultados superiores aos do presente estudo foram encontrados por Nathan et al. (2006), ao observarem 
um declínio próximo a $90 \%$ na taxa de eclosão de ovos do díptero, Anopheles stephensi tratados com o extrato de sementes de Melia azedarach. Abdel-Shafy e Zayed (2002) observaram, ao tratar ovos do carrapato Hyalomma anatolicum excavatum, um efeito deletério significante sobre o embrionamento dos ovos com o composto Neem-Azal F com taxas de eclosão variando de 34 a $60 \%, 15$ dias após o tratamento.

Em relaçáo ao efeito larvicida, $67,75 \pm 2,21 \%$ das larvas não chegaram à fase de pupa. $\mathrm{A} \mathrm{CE}_{50}$ verificada nestes estudos para as larvas foi de $60,98(45,93-91,62) \mathrm{mg} \cdot \mathrm{mL}^{-1}$. Eficácias superiores a este trabalho foram descritas, quando OKUMU et al. (2007) testaram o óleo da semente do nim sobre larvas de Anopheles gambiae e obtiveram uma $\mathrm{CE}_{50}$ de 10,7 ppm. De forma semelhante, menor eficácia foi observada por RAHUMAN et al. (2008) com o acetato de gluanol, um triterpeno tetracíclico, sobre as larvas dos mosquitos Aedes aegypti, Culex quinquefasciatus e A. stephensi. Esses autores obtiveram $\mathrm{CE}_{50}$ de 14,55; 41,42 e 28,50 ppm para as três espécies, respectivamente. Poucos são os estudos realizados com flebotomíneos; no entanto, COELHO et al. (2006) adicionaram azadiractina à dieta de larvas de $L$. longipalpis, em laboratório, verificando que, nas concentraçóes de 0,$1 ; 1,0$ e $10,0 \mu \mathrm{g}$ de azadiractina. $\mathrm{mg}^{-1}$ de dieta, houve um aumento significante da mortalidade larvar. Concentraçôes de 0,1 e 1,0 $\mu \mathrm{g}$ de azadiractina. $\mathrm{mg}^{-1}$ na dieta bloquearam a muda das larvas do inseto, que permaneceram no terceiro estágio até o final do experimento.

No ensaio com adultos, na concentração de $100 \mathrm{mg} \cdot \mathrm{mL}^{-1}$, observou-se uma mortalidade de 96,64 $\pm 4,11 \%$ dos insetos após 24 horas de exposição ao óleo de nim. Resultados inferiores a este trabalho foram obtidos por Luitgards-Moura et al. (2002), ao testar os extratos aquosos de Antonia ovata e Derris amazonica sobre adultos de $L$. longipalpis, verificando, após 48 horas de observação, $\mathrm{CL}_{50}$ de 233 e $212 \mathrm{mg} \cdot \mathrm{mL}^{-1}$, respectivamente. Também Nathan et al. (2006) obervaram menor percentual de mortalidade $(74,6 \pm 6,2 \%$ e $90,9 \pm 7,0 \%$ respectivamente), de adultos do inseto sobre $A$. stephensi tratados com os extratos metanólicos de folhas e sementes de Melia azedarach.

Neste trabalho, a análise fitoquímica do óleo de sementes de $A$. indica revelou a presença de triterpenos. Os terpenos abrangem uma grande variedade de substâncias de origem vegetal, e sua importância ecológica como defensivos de plantas está bem estabelecida, podendo ser classificados, basicamente, em monoterpenos, sesquiterpenos, diterpenos e triterpenos; dentre esses últimos, estão os limonoides (VIEGAS-JÚNIOR, 2003). Existe uma grande diversidade de limonoides isolados da família Meliaceae, entre eles azedarachinas, sendaninas e trichilinas, além dos que apresentam o anel C-seco, como a azadiractina que é o principal composto. Estes podem ser encontrados em todos os tecidos das plantas;, no entanto, os órgáos podem, individualmente, produzir diferentes tipos de limonoides (NAKATANI et al., 1995).

O nim é capaz de se proteger contra grande número de pragas em razão da variedade de uma grande quantidade de compostos bioativos. Seus principais elementos químicos incluem uma mistura de 3 ou 4 compostos correlatos, que podem ser modificados em mais de 20 outros menores, porém náo menos ativos. No geral, esses compostos pertencem à classe dos produtos naturais conhecidos por triterpenos, mais especificamente limonoides. De fato, pelo menos 9 limonoides de nim têm demonstrado habilidade em bloquear o desenvolvimento de pragas agrícolas (MOSSINI; KEMMELMEIER, 2005).
Um grande número de novos triterpenoides com atividade biológica vem sendo isolados de extratos de sementes e folhas do nim (LUO et al., 1999). Também têm sido identificados produtos derivados e produtos análogos do composto azadiractina (RAMJI et al., 1998). Dos nove isômeros de azadiractina descritos na literatura, azadiractina A e B representam a maior concentração de metabólitos presentes nas sementes e são consideradas importantes para a comercialização da planta, como biopesticida (SIDHU et al., 2003). O composto azadiractina A de $A$. indica, e um grupo de outros limonoides estâo intimamente associados à ação supressora de apetite ou inibidora de crescimento em insetos e têm sido extensamente estudados, com o objetivo de conhecer a química, biossíntese, toxicologia e o potencial inseticida desse grupo de compostos naturais (VIEGAS-JÚNIOR, 2003). A azadiractina concentra-se principalmente nos frutos, aumentando ao longo do desenvolvimento, sendo máxima no amadurecimento e durante o armazenamento, podendo sofrer variaçóes de acordo com o modo de colheita, armazenamento, teores de umidade, presença de luz e temperatura (SCHMUTTERER, 1990). Essa substância, não necessariamente, age matando diretamente o inseto, como a maioria dos inseticidas; atua sobre a reprodução e crescimento ou como toxina direta (ROY; SARAF, 2006). Ao que se sabe, um dos mecanismos de ação mais comum da azadiractina é por meio da inibição da síntese de quitina necessária para o desenvolvimento dos insetos (CASSIER; PAPILLON, 1991).

\section{Conclusão}

Diante dos resultados obtidos, conclui-se que o óleo das sementes de $A$. indica possui atividade inseticida sobre as três fases de L. longipalpis em laboratório. Esses resultados podem indicar que compostos originários de plantas, como os limonoides presentes no nim, podem ser uma alternativa aos inseticidas químicos no controle do vetor da LV. Outros estudos devem ser realizados para comprovar a atividade das substâncias isoladas sobre o inseto.

\section{Referências}

ABDEL-SHAFY, S.; ZAYED, A. A. In vitro acaricidal effect of plant extract of neem seed oil (Azadirachta indica) on egg, immature, and adult stages of Hyalomma anatolicum excavatum (Ixodoidea: Ixodidae). Veterinary Parasitology, v. 106, n. 1, p. 89-96, 2002.

ALMEIDA, J. E. M.; FILHO, A. B. Banco de microrganismos entomopatogênicos. Biotecnologia Ciência e Desenvolvimento, n. 20 , p. 30-33, 2001.

BEJARANO, E. E. et al. Phlebotomine sand flies (Diptera: Psychodidae) associated with the appearance of urban leishmaniasis in the city of Sincelejo, Colômbia. Memórias do Instituto Oswaldo Cruz, v. 97, n. 5, p. 645-647, 2002.

BOEKE, S. J. et al. Safety evaluation of neem (Azadirachta indica) derived pesticides. Journal of Ethnopharmacology, v. 94, n. 1, p. 25-41, 2004.

CASSIER, P.; PAPILLON, M. Ultrastructural studies of the in vitro chitin and cuticle secretions in the wig pads of Locusta. Canadian Journal of Zoology, v. 69, n. 1, p. 29-38, 1991. 
COELHO, C. A. A. et al. Effects of azadirachtin on the development and mortality of Lutzomyia longipalpis larvae (Diptera: Psychodidae: Phlebotominae). Journal of Medical Entomology, v. 43, n. 2, p. 262-266, 2006.

COSTA, C. H. N. Characterization and speculations on the urbanization of visceral leishmaniasis in Brazil. Cadernos de Saúde Pública, v. 24, n. 12, p. 2959-2963, 2008.

DANTAS-TORRES, F.; BRANDÃO-FILHO, S. P. Expansão geográfica da leishmaniose visceral no Estado de Pernambuco. Revista da Sociedade Brasileira de Medicina Tropical, v. 39, n. 4, p. 352-356, 2006.

DESJEUX, P. Leishmaniasis: current situation and new perspectives. Comparative Immunology, Microbiology \& Infectious Diseases, v. 27 , n. 5, p. 305-318, 2004.

GALLO, D. et al. Entomologia agrícola. Piracicaba: FEALQ, 2002. $920 \mathrm{p}$.

GONTIJO, C. M. F.; MELO, M. N. Leishmaniose Visceral no Brasil: quadro atual, desafios e perspectivas. Revista Brasileira Epidemiologia, v. 7 , n. 3, p. 338-349, 2004.

LUITGARDS-MOURA, J. F. et al. Preliminary assays indicate that Antonia ovata (Loganiaceae) and Derris amazonica (Papilionaceae), ichthyotoxic plants used for fishing in Roraima, Brazil, have an insecticide effect on Lutzomyia longipalpis (Diptera: Psychodidae: Phlebotominae). Memórias do Instituto Oswaldo Cruz, v. 97, n. 5, p. 737-742, 2002.

LUO, X. et al. Two novel azadirachtin derivatives from Azadirachta indica. Journal of Natural Products, v. 62, n. 7, p. 1022-1024, 1999.

MATIAS, R. et al. Melia azedarach, uso popular x estudos químico e farmacológico: breve revisão. Ensaios e Ciência, v. 6, n. 1, p. 91-121, 2002.

MATOS, F. J. A. Introduçáo à fitoquímica experimental. 2 ed. Fortaleza: UFC, 1997. 141 p.

MOSSINI, S. A. G.; KEMMELMEIER, C. A árvore Nim (Azadirachta indica. A. Juss.): múltiplos usos. Acta Farmaceutica Bonaerense, v. 24, n. 1, p. 139-148, 2005.

NAKATANI, M. et al. Phragmalin limonoids from Chukrasia tabularis. Phytochemistry, v. 65, n. 20, p. 2833-2841, 2004.

NAKATANI, M. et al. Salannal, a new limonoid from Melia azedarach Linn. Chemistry Letters, v. 24, n. 11, p. 995, 1995.
NATHAN, S. S. et al. Efficacy of Melia azedarach L. extract on the malarial vector Anopheles stephensi Liston (Diptera: Culicidae). Bioresource Technology, v. 97, n. 11, p. 1316-1323, 2006.

OKUMU, F. O.; KNOLS, B. G. J.; FILLINGER, U. Larvicidal effects of a neem (Azadirachta indica) oil formulation on the malaria vector Anopheles gambiae. Malaria Journal, v. 6, p. 63, 2007.

PASCUAL-VILLALOBOS, M. J.; ROBLEDO, A. Screening for antiinsect activity in Mediterranean plants. Industrial Crops and Products, v. 8, n. 3, p. 183-194, 1998.

RAHUMAN, A. A. et al. Mosquito larvicidal activity of gluanol acetate, a tetracyclic triterpenes derived from Ficus racemosa Linn. Parasitology Research, v. 103, n. 2, p. 333-339, 2008.

RAMJI, N.; VENKATAKRISSHNAN, K.; MADYASTHA, K. M. 11-epi-azadirachtin D: An epimeric azadirachtin analogue from Azadirachta indica. Phytochemistry, v. 49, n. 1, p. 265-267, 1998.

ROY, A.; SARAF, S. Limonoids: overview of significant bioactive triterpenos distributed in plants kingdom. Biological Pharmaceutical Bulletin, v. 29, n. 2, p. 191-201, 2006.

SCHMUTTERER, H. Properties and potential of natural pesticides from the neem tree, Azadirachta indica. Annual Review of Entomology, v. 35, p. $271-297,1990$.

SHERLOCK, I. A.; SHERLOCK, V. A. Métodos práticos para criação de flebotomíneos em laboratório. Revista Brasileira de Biologia, v. 32, n. 2, p. 209-217, 1972.

SIDHU, O. P.; KUMAR, V.; BEHL, H. M. Variability in neem (Azadirachta indica) with respect to azadirachtin content. Journal of Agricultural and Food Chemistry, v. 51, n. 4, p. 910-915, 2003.

SINGH, S. New developments in diagnosis of leishmaniasis. Indian Journal Medicinal Research, v. 123, n. 3, p. 311-330, 2006.

TARE, V.; DESHPANDE, S.; SHARMA, R. N. Susceptibility of two different strains of Aedes aegypti (Diptera: Culicidae) to plant oils. Journal of Economic Entomology, v. 97, n. 5, p. 1734-1736, 2004.

VIEGAS JÚNIOR, C. Terpenos com atividade inseticida: uma alternativa para o controle químico de insetos. Química Nova, v. 26, n. 3, p. 390-400, 2003. 